\title{
Corrigendum à : Little Boney, grosse menace : Une analyse de l'image [2(2), (2017), 202-214]
}

\author{
${ }^{1}$ Witakania SUNDASARI, ${ }^{2}$ Ferli HASANAH \\ ${ }^{1}$ Université de Poitiers - France/ Universitas Padjadjaran - Indonésie ; \\ ${ }^{2}$ Universitas Padjadjaran, Indonésie
}

Reçu le 10 février 2018 | Évalué le 11 février 2018 | Accepté le 12 février 2018

CORRIGENDUM À : Sundasari et Hasanah, (2017). Francisola : Revue Indonésienne de la
langue et la littérature françaises, 2(2), 202-214. dx.doi.org/10.17509/francisola.v2i2.9412).

Dans les coordonnées des auteurs, l'établissement de rattachement du premier auteur est écrit «Université de Poitiers - France» et le courriel du premier auteur est écrit «sundasari.som@univ-poitiers.fr».

Dans les coordonnées des auteurs, il faudrait ajouter l'établissement de rattachement du premier auteur, qui est «Universitas Padjadjaran - Indonésie », et le courriel du premier auteur, qui est «witakania.som@unpad.ac.id ».

ERRATUM : Sundasari et Hasanah, (2017). Francisola : Revue Indonésienne de la langue et la littérature françaises, 2(2), 202-214. (doi: dx.doi.org/10.17509/francisola.v2i2.9412).

Dans les coordonnées des auteurs, l'établissement de rattachement du premier auteur, qui est «Universitas Padjadjaran - Indonésie », et le courriel du premier auteur, qui est «witakania.som@unpad.ac.id », ont été ajoutés.

Par conséquent, les coordonnées de l'établissement de rattachement et du courriel du premier auteur ont été corrigées comme suit: «Université de Poitiers - France/ Universitas Padjadjaran - Indonésie » et «sundasari.som@univpoitiers.fr/witakania.som@unpad.ac.id»

Mots-clés : analyse d'image, angleterre, caricature, révolution française.

画 auteur correspondant : sundasari.som@univ-poitiers.fr/witakania.som@unpad.ac.id

Pour citer cet article [Style APA] : Sundasari et Hasanah, (2017). Corrigendum à : Little Boney, grosse menace : Une analyse de l'image [2(2), (2017), 202-214]. Francisola: Revue Indonésienne de la langue et la littérature françaises, 3(1), 95. doi: 10.17509/francisola.v3i1.11895 